\title{
The efficacy of cognitive rehabilitation therapy: A meta-analytic review of traumatic brain injury and stroke cognitive language rehabilitation literature
}

\author{
Zachary Miklos, Matthew Mychailyszyn, Rick Parente \\ Department of Psychology, Towson University, Towson, U. S. A. \\ Email address: \\ mikloszr@gmail.com (Zachary M.),mmychailyszyn@towson.edu (Matthew M.),rparente007@yahoo.com (Rick P.)
}

\section{To cite this article:}

Zachary Miklos, Matthew Mychailyszyn, Rick Parente. The Efficacy of Cognitive Rehabilitation Therapy: A Meta-Analytic Review of Traumatic Brain Injury and Stroke Cognitive Language Rehabilitation Literature. American Journal of Psychiatry and Neuroscience. Vol. 3, No. 2, 2015, pp. 15-22. doi: 10.11648/j.ajpn.20150302.11

\begin{abstract}
Traumatic brain injury (TBI) and stroke are leading contributors to health impairments and decrements to quality of life. This meta-analysis evaluated 10 studies of cognitive language rehabilitation and recovery among patients who endured a traumatic brain injury (TBI) or stroke. Results indicated a significant effect size in the control conditions $(r=.27 p<.05)$ in which patients received no cognitive language rehabilitation therapy, suggesting that considerable language improvement occurs spontaneously over time. A significant effect size was also present in the intervention conditions $(r=.4, p<.05)$ in which patients received cognitive language rehabilitation therapy. There was a significant difference between these two effects sizes, which suggests that cognitive language rehabilitation therapies are effective therapeutic interventions above and beyond what can be contributed solely to the passage of time.
\end{abstract}

Keywords: Brain Injury, Stroke, Language, Rehabilitation, Meta-Analysis

\section{Introduction}

According to the Centers for Disease Control (CDC), traumatic brain injury (TBI) is a contributing factor to $30.5 \%$ of all injury-related deaths in the United States [1]. In sporting contexts alone, it has been reported that between 300,000 to 1.54 million mild-to-moderate brain injuries occur per year [2]. Troublingly, the pathophysiology of this injury is complex. $\mathrm{Wu}$ et al. [3] stated that the pathophysiology of TBI can involve damage that occurs at the time of injury (primary) and damage that may not appear clinically for hours or days after the injury (secondary). While there are many causes of TBI and mild traumatic brain injury (mTBI), McNally and colleagues [4] suggest that the outcomes associated with these injuries are poorly understood. Because the brain injury population is large and growing, rehabilitation therapies have proliferated in areas such as memory, language, attention, and emotional disturbances. Research concerning the efficacy and effectiveness of these treatments, has produced mixed results.

The Brain Injury Association of America [5] characterizes treatment for brain injury as a continuum of care in which individuals may enter, exit, and re-enter treatment at any point during the course of their recovery. It is, therefore, necessary to assess which treatments are effective at each stage of a person's recovery. It is also necessary to assess which treatments are effective in treating a multitude of cognitive disorders, such as, stroke, dementia, or severe developmental delays. The following discussion will focus on research regarding the symptoms and treatment of stroke, as the features of this disorder are most similar to those of TBI.

\subsection{Introduction to Stroke and Aphasia}

Stroke is one of the main causes of mortality and disability in industrialized countries [6]. On average, one United States citizen dies from a stroke every four minutes [7]. Most stroke victims are single, live at home, and require more in-home assistance than in previous decades [8].

Aphasia is one of the most common and enduring neurological outcomes after stroke; this outcome remains acute in $30-45 \%$ of post-stroke aphasic individuals [9]. Relative to TBI literature, there is a plethora of research on aphasia; however, westernized countries appear to be overrepresented in this literature, as Beveridge and Bak [10] state that $62 \%$ of this body of research involves English 
speaking aphasic patients and less than $8 \%$ of aphasia-related research relates to non-Indo-European languages.

Further, stroke patients present with heterogeneous symptoms including depression [11], movement disorders [12], physical impairment and fatigue [13] and a variety of language-related disorders. The recovery process usually includes environmental, motoric, and/or somatosensory enrichment that is designed to capitalize on the brain's neuroplasticity [14]. Because of this diversity of symptoms, treatments for stroke are varied.

\section{Cognitive Language Rehabilitation}

The results of several studies $[15,16,17]$ have led to endorsement of individualized therapies that offer specific treatment outcomes, are designed for specific clinical populations, and are focused on facilitating recovery of a variety of brain mechanisms. Diversity of treatment is necessary because of the equally diverse behavioral and/or cognitive abnormalities that can arise after stroke or TBI. While Broca's and Wernicke's areas are the brain regions most often investigated regarding language deficits in stroke and brain injury $[18,19,20,21]$, these are not the only neuroanatomical regions that can affect language. Grey and white matter [22], the left inferior frontal cortex [23], and the peri-Sylvian area [24] also determine language skill and are often affected after stroke or TBI.

\subsection{Cognitive Language Rehabilitation Efficacy}

The efficacy of cognitive rehabilitation therapy after brain injury or stroke has been a topic of interest in a number of reviews on treatment efficacy [25, 26, 27, 28, 29]. Cicerone et al. [25] systematically reviewed 171 articles that assessed the efficacy of numerous cognitive rehabilitation interventions. These articles were broken into seven categories that reflected the articles' primary intervention focus: attention, visual perception and constructional abilities, language and communication, memory, problem solving and executive functioning, along with a wide variety of multi-modal interventions, and several comprehensive-holistic cognitive rehabilitations.

Cicerone and colleagues [25] found strong evidence that a cognitive rehabilitation intervention facilitated recovery in individuals with TBI and stroke. Regarding language treatments, the authors noted two points: 1) that interventions directed at improving pragmatic communication and conversational skills were especially efficacious for TBI survivors and 2) that interventions targeted at reading comprehension and language formation were useful with left hemisphere stroke or TBI survivors [25].

Similar to Cicerone et al. [25, 26] and Robey [27], Rohling et al. [28] evaluated 115 distinct studies that were cited in both of the former reviews. Rohling et al. [28] included 70 single-group pre-post (SGPP) designs and 45 independent groups pre-post (IGPP) designs. Exclusionary criteria used by Rohling et al. [28] (but not Cicerone et al. [25, 26]) included removal of single-case studies or multiple case studies with a total sample size less than three $(\mathrm{N}<3)$ and studies that did not provide sufficient statistical information to calculate the mean difference. Rohling and colleagues [28] also investigated potential moderator variables such as etiology, recovery level, and the mean age of patient samples. The authors concluded that: a) cognitive rehabilitation for attention, visuospatial, and language produced significant improvements, b) memory treatments produced vague results, and c) comprehensive treatments failed to produce a significant improvement.

Regarding cognitive language treatment efficacy, Rohling et al. [28] found significant effect sizes for SGPP's $(p<.05)$ and IGPP's $(\mathrm{p}<.01)$. However, some of these included in Rohling et al. [28] discussed post-TBI language treatments, leading the authors to conclude that while the language therapies effect size was significant, there was not "adequate quantitative basis to support all of the general practice guidelines suggested by the systematic review" (p. 33). While Cicerone et al. [25] claimed that there is a strong body of evidence supporting the benefit of language therapies for TBI and stroke, Rohling et al. [28] found weak support for this claim, however, stating that it is difficult to make a conclusive argument about the benefit of cognitive language rehabilitation until more treatments are developed to treat language deficits and more studies are done to evaluate these treatments.

The purpose of the present study was, therefore, to evaluate the efficacy of cognitive language rehabilitation in contemporarily published studies not represented in the Rohling et al. [28] meta-analysis. A precedent for such an endeavor has been set by earlier investigators: Specifically, Elliot and Parente [30] sought to update the findings yielded by Rohling and colleagues by conducting a meta-analysis of memory rehabilitation interventions following stroke and TBI. Additionally, Bernard [31] conducted a meta-analysis of attention rehabilitation interventions as an update to Rohling and colleagues.

\section{Meta-Analysis and Effect Size Measures}

Meta-analysis is an approach to the statistical aggregation and evaluation of a collection of previously conducted studies for the purpose of integrating the findings. The Task Force on Statistical Inference of the American Psychological Association emphasizes, "that reporting and interpreting effect sizes in the context of previously reported effects is essential to good research" [32, p. 599]. The goal of a meta-analysis is generally to draw conclusions about what is currently known about a topic or issue by assessing the overall effect sizes of specific variables examined by formerly conducted studies in the corresponding area. The effect size is the magnitude and direction of the difference between two groups or the relationship between two variables [33]. Effect sizes provide an assessment of the shared correlation between independent and dependent variables. Homogenous collections of effect sizes indicate consistent relationships 
across studies [28].

\section{Methods}

The present study is an updated meta-analytic review of cognitive language rehabilitation studies for brain injury and stroke that are more recent than those previously reviewed by Rohling et al. [28]. Studies pertaining to cognitive language rehabilitation efficacy were indexed by Medline, PubMed, PsycINFO, and Google Scholar and selected using the following keywords: "lang*," "cognitive," "rehab*," "traumatic brain injury," "TBI," "stroke," "aphas*," and "treatment."

\subsection{Exclusion Criteria}

Studies were excluded based on Rohling et al.'s [28] criteria: a) articles not investigating cognitive language rehabilitative interventions, b) articles that merely describe - rather than evaluate - treatment approaches or theories, c) other review articles, d) unspecified or unmeasured interventions, e) articles lacking diagnosis or assessment of TBI or stroke, f) case studies of a single participant with no empirical data, g) non-peer reviewed articles, h) articles including exclusively-pharmacological interventions, and i) articles not written in English. Additional criteria for exclusion not used by Rohling and colleagues [28] included articles that did not present the means or standard deviations for groups. In total, ten published cognitive language rehabilitation studies met selection standards and were included in the analysis. All of the studies had an intervention group. Six of the studies compared a control group to an intervention group.

\subsection{Procedural Analysis}

Effect sizes were computed from the available statistics in each of the published studies. These values (calculated as correlations in the form of $r$ ) were analyzed to determine if the aggregate effect sizes were significantly different from zero. The authors used Pearson's r, as it is one of the most universally applicable indexes of effect size [34]. The first analysis concerned the control conditions' effect sizes and estimated to what extent language improvement could be attributable to the passage of time. The second analysis concerned the intervention conditions' effect sizes and estimated how much language improvement could be attributed to cognitive rehabilitation therapy and with the passage of time. A third analysis explored the difference between the control and experimental group effect sizes in order to assess whether cognitive language rehabilitation interventions yielded significantly larger effect sizes compared to what could be attributed to the passage of time, as reflected by the control conditions. A fourth analysis compared the effect size differences of the control and intervention conditions to those reported by Rohling et al. [28]. The homogeneity of the collection of effect sizes was assessed through the use of forest plots portraying study effect sizes. The horizontal lines that pass through the data points in each plot represent the confidence interval for that effect size. The extent to which the horizontal lines overlap provides an indication of the homogeneity of the effect sizes across studies. Finally, Begg's test statistic was calculated to assess publication bias, or the extent to which the studies overestimate the effect size.

\section{Results}

\subsection{Improvement Due to the Passage of Time}

The average effect size for the control conditions across studies was significant $(\mathrm{r}=.27, \mathrm{p}<.05)$, CIs $[.21, .32])$, indicating a significant improvement in language over time, without direct intervention. Fig. 1 and 2 display forest plots of the effect sizes, expressed as correlations, for all included studies in control and intervention conditions, respectively. The forest plots in Fig. 1 and 2 show that the conditions were generally homogeneous.

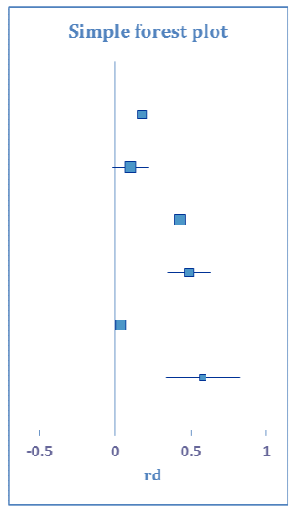

Figure 1. Forest plot of effect sizes and confidence intervals in control group studies.

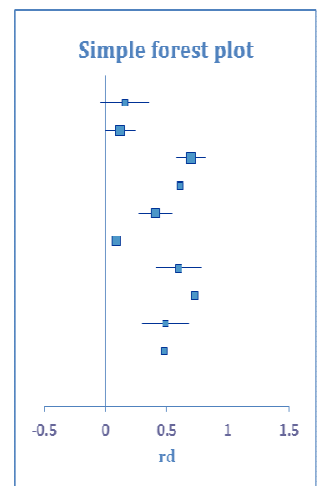

Figure 2. Forest plot of effect sizes and confidence intervals in intervention group studies.

Intervention conditions showed a significant average effect $\operatorname{size}(r=.40, p<.05)$, CIs $[.34, .45]$, indicating that significant improvement occurs amongst individuals receiving cognitive language rehabilitation.

\subsection{Comparison to Rohling et al. [28]}

Overall, the results of this study are similar to Rohling et al. [28]. Both Rohling et al. [28], $\mathrm{r}=.3, \mathrm{p}<.05$, CIs [.24, .35], and 
the present study found that some amount of language improvement could be attributed to the passage of time. Forest plots in Fig. 3 and 4 display effect sizes, expressed as correlations, for all included control and cognitive language intervention studies in Rohling et al. [28]. As is the case in this study, the forest plots of the Rohling et al. [28] review suggests that the studies were also generally homogeneous.

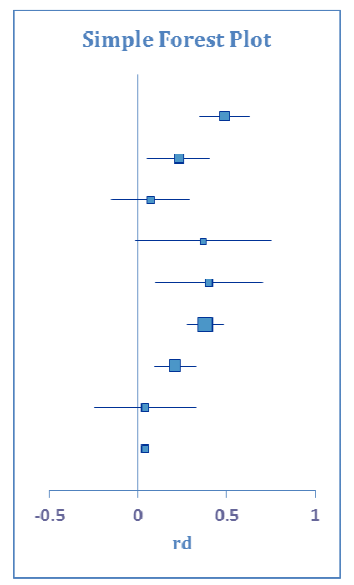

Figure 3. Forest plot of effect sizes and confidence intervals in Rohling et al. (2009)'s cognitive language control group studies.

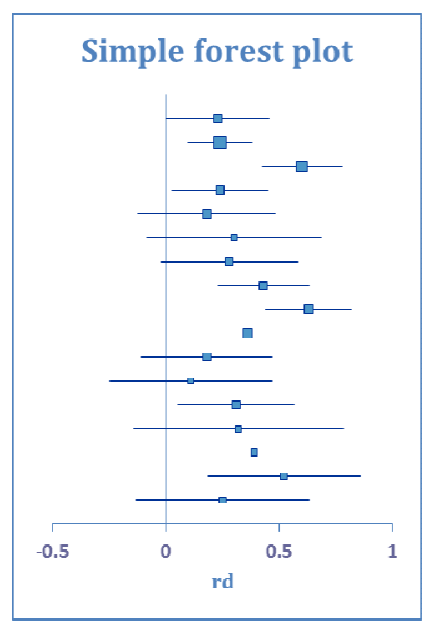

Figure 4. Forest plot of effect sizes and confidence intervals in Rohling et al. (2009)'s cognitive language intervention group studies.

The confidence interval associated with the effect sizes in the present study contained the average effect size reported by Rohling et al.[28]. Although the average effect size was somewhat higher in the present study compared to Rohling's et al.'s [28] original language effect size, there was no significant difference found between the two, $\mathrm{p}>.05$. However, in both reviews, the aggregate effect sizes across intervention conditions were significantly larger than the aggregate effect sizes obtained from the control groups of studies that utilized such conditions. This difference suggests that the experimental treatments produced significant improvements in language functioning. This effect was originally documented in Rohling et al. [28] and replicated here.

\subsection{Difference Between the Control and Intervention Groups}

Because the confidence interval for the intervention conditions effect size, CIs $[.34, .45]$, did not contain the effect size of the control condition, 27, this indicates that some amount of improvement could not be attributed to the passage of time. The difference between these effect sizes suggests that 13 percent of the effect that occurs in intervention studies results from the intervention itself whereas the remainder of the effect size results from spontaneous change.

\subsection{Publication Bias}

Begg's Test statistic was computed on the control conditions' effect sizes in order to determine whether publication bias affected the results. The Begg's Test statistic was not significant $(\mathrm{p}=.85)$ indicating that publication bias was not present in the studies. Funnel plots, as depicted in Fig. 5 and 6 , did not indicate any dissemination bias, as suggested by the random distribution of points around the synthesis estimate line. Fig. 6 also shows a lack of publication bias in Rohling et al.'s [28] studies, as well $(\mathrm{p}=.59)$.

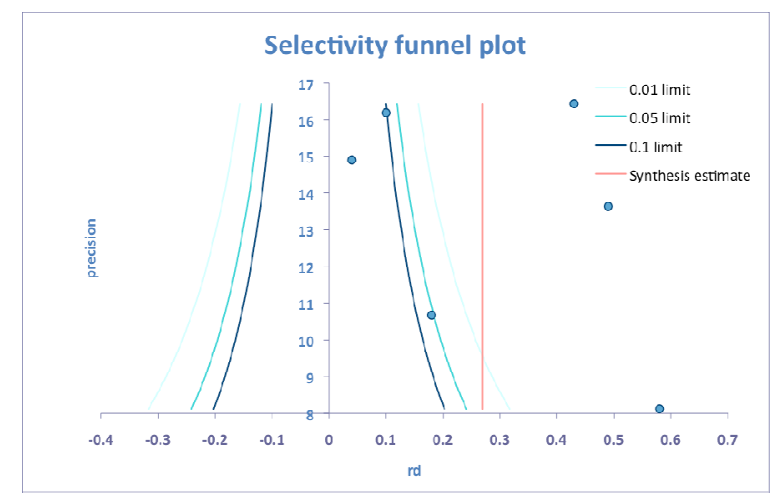

Figure 5. Funnel plot for language control group studies. This figure illustrates the homogeneity of effect sizes for control group studies.

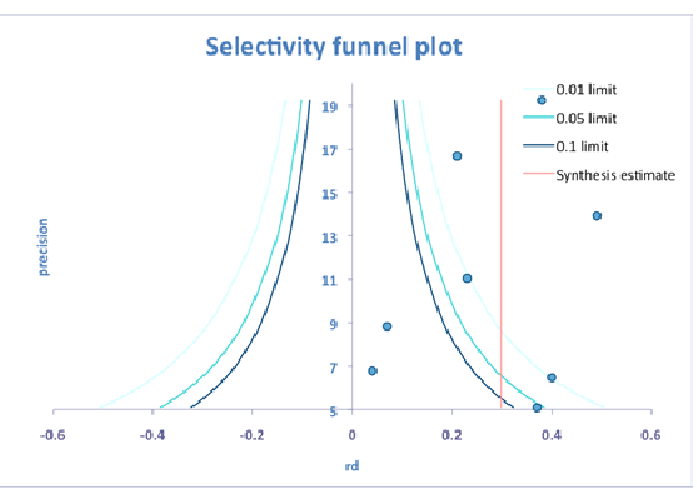

Figure 6. Funnel plot for Rohling et al. (2009)'s cognitive language control group studies. This figure illustrates the homogeneity of effect sizes for Rohling et al. (2009)'s control group studies.

Begg's Test statistic was also computed on the intervention conditions' effect size and found to be not significant $(p=.53)$ indicating that publication bias was not present in the current set of studies. Fig. 7 indicates a lack of publication bias, as 
there is a random distribution of studies around the synthesis estimate line. This same pattern is present in Rohling et al.'s [28] studies as well ( $p=.90$, see Fig. 8).

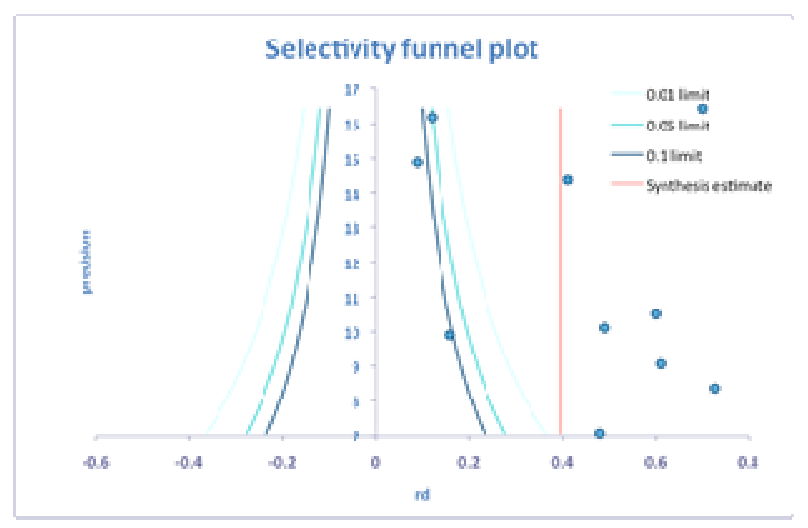

Figure 7. Funnel plot for intervention group studies. This figure illustrates the homogeneity of effect sizes for the intervention group studies.

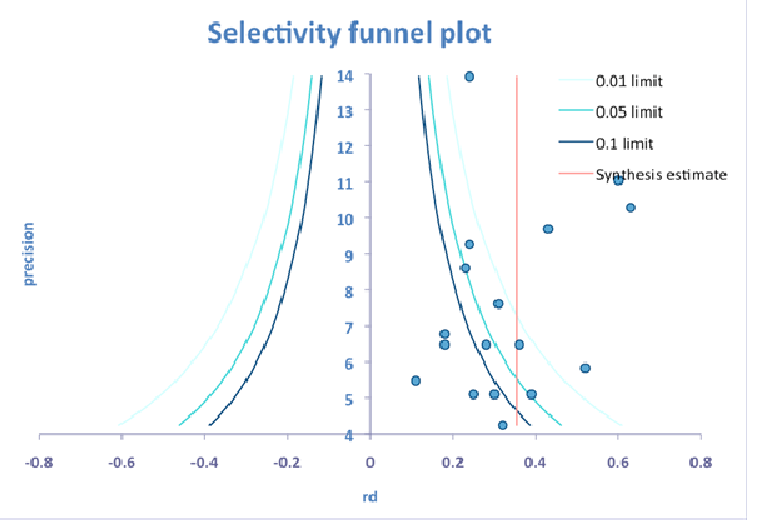

Figure 8. Funnel plot for Rohling et al. (2009)'s cognitive language intervention group studies. This figure illustrates the homogeneity of effect sizes for Rohling et al. (2009)'s cognitive language intervention group studies.

\section{Discussion}

The present meta-analysis obtained results that are generally consistent with the findings of Rohling and colleagues [28], with contemporary studies not represented in their analysis. Both reviews show a significant effect that occurs with the passage of time; they also show an effect size for the intervention groups that was significantly larger than that found in the control conditions.

We also performed a post hoc comparison between the effect sizes for the stroke versus TBI studies. This analysis showed that the effect size for stroke patients is somewhat larger than that for TBI survivors which is consistent with other meta-analyses that showed similar differences in these patient populations [31, 30]. This difference may be due to: a) the cognitive language rehabilitations for brain injury and stroke having different methodologies, b) the factors that influence language deficits in brain injury and stroke being different and, therefore, having different interventions that would work effectively and efficaciously, and/or c) cognitive language rehabilitations for stroke being more developed than those for TBI.

Another consistency concerns the lack of publication bias in these data and in Rohling et al.'s [28] published work. There is little evidence of unpublished data that would undermine these findings. Although it is certainly possible that less effective interventions might produce smaller effect sizes, these differences should be investigated with ongoing meta-analytic procedures that code for the type of intervention. Regarding the set of interventions that were used in these studies, the effects are clear: while most of the improvement occurred spontaneously, language interventions produced an effect that was larger than what occurred without intervention. Therefore, although patients may improve spontaneously, these study findings provide evidence that clinical intervention may significantly accelerate the patients' recovery.

\subsection{Limitations}

There are several limitations to the conclusions derived from this or any other meta-analysis of studies pertaining to cognitive functions. The first involves a general dearth of studies meeting inclusion criteria. The data that are available largely derive from pre-existing records, practice files of a single therapist, case studies, and self-report. There are few clinical trials and even fewer studies that contain appropriate control groups as ethical considerations make many experimental manipulations, including withholding treatment, unfeasible. Therefore, for a variety of valid reasons, the ratio of well controlled to quasi-experimental studies is small and thus a limited number of studies met the inclusion criteria for this meta-analytic investigation.

While there are numerous articles that discuss language therapies, there is not a wealth of those that specifically focus on cognitive rehabilitation therapies regarding language. The present study could have included more studies if inclusion criterion were less stringent; however, the authors opted to draw conclusions from a smaller number of more methodologically rigorous studies rather than to risk conclusions that were based on a larger number of studies with limited internal validity. However, the absence of publication bias in the present analysis reflects that our decision to apply this strategy did not lead to spurious conclusions; the consistency of conclusions drawn from this review along with those of Rohling and colleagues [28] suggests that the effect sizes seem to be relatively stable over time.

These results are also limited to studies of patients with two types of disability, stroke and brain injury. However, language disorders result from a number of maladies, including but not limited to, dementia, schizophrenia, developmental disorders, and progressive degenerative diseases. It is, therefore, likely that the effects of cognitive language rehabilitation therapy might differ in relation to the nature of the patient's unique medical condition. Because this study chose to provide an update of Rohling et al. [28], only individuals with brain injury and stroke were included.

A further limitation is that research in this field lacks formalized criteria regarding the extent of the brain injury or stroke. The exception to this statement is the definition of concussion, i.e., a mild brain injury. In 1997, the Quality Standards Subcommittee defined a concussion as "any trauma 
induced alteration in mental status that may or may not include a loss of consciousness" [35, p. 582]. However, beyond concussion, such well-defined descriptions are not available. Often a patient's injuries are complicated by a variety of ancillary disorders, psychiatric problems, varying medication regimens, or predisposing conditions that all coalesce to limit their cognitive functioning and delay their recovery. These problems are seldom discussed in research publications and make it difficult to match patient populations in future studies. Lack of formalized definitions such as this cause variation in clinician definitions of injury types.

A final limitation is that there is no consistent definition of cognitive language rehabilitation therapy, which makes it difficult for interested investigators to replicate treatment interventions. Even in well-controlled studies, it is often difficult to determine exactly how the treatment was administered. Unlike modalities of treatments in other disciplines, cognitive language rehabilitative therapies often do not have standardized procedures for implementation. Instead, most cognitive language rehabilitation therapies involve vague descriptions of interventions that are difficult to replicate. It is very challenging to accurately determine what does and what does not constitute a cognitive rehabilitation therapy. There is a lack of standardized treatments that can be evaluated for efficacy. This issue is part of a larger problem in the field of cognitive rehabilitation therapy. In order for future meta-analyses to be run with methodologically rigorous criteria, the field of cognitive rehabilitation needs to define in detail what makes a rehabilitation therapy a cognitive rehabilitation therapy.

The authors present a possible definition for cognitive language rehabilitation therapy as: a treatment modality that specifically addresses language deficits, whether resulting from organic or inorganic brain damage, through interventions focused on developing synapses to promote language recovery. While it is unfeasible for clinicians to mandate their patients undergo baseline and post-intervention imaging to measure synaptic growth, it can be assumed that synaptic growth has occurred if language improvement is occurring, i.e. neuroplasticity. This process is commonly thought to be what is occurring neurologically when language function increases following brain damage [36].

It is important to note that cognitive rehabilitation programs that address a variety of cognitive domains are not necessarily language based cognitive rehabilitation therapy. Effective therapies for language rehabilitation must have a clear rational and evidence base [37, 38, 39, 40, 41]. Many of the articles that were rejected from this meta-analysis involved global programs of therapy that had a positive effect on language functioning. Although these global program based therapies may improve language functioning, they do not target specific language functions nor do they demonstrate a causal relationship between specific treatments for language disorders and improve language.

\subsection{Recommendations for Future Research}

It is reasonable to suggest that an ongoing update of the data from published studies should be undertaken to continually study changes in the field and to evaluate the efficacy of interventions. As a step in this direction, the present data base $[14,38,39,40,41,42,43,44,45,46]$ is available on demand from the authors. It is our hope that other researchers will add to it and draw their own conclusions from it. Consistently updating a shared database would provide all researchers with the most accurate and easily accessible data for an ongoing meta-analysis of cognitive language rehabilitation research.

Reviewing the extant literature in this topical area suggested a need to develop consistent criteria for cognitive language rehabilitation interventions along with standardized treatment batteries that can be applied consistently across studies. An example of such a standardized treatment is the Attention Process Training program developed by Sohlberg and Mateer [37], which has demonstrated efficacy and can be used as a standardized model for training attention.

It is also important to evaluate different covariates that may influence treatment efficacy. A litany of covariates may influence the outcome of cognitive rehabilitation treatments, including the type of disorder, patient history, predisposing conditions, education, medication regimen, the extent of the patients' family support structure, ability to live independently, and the consistency of therapy after TBI or stroke. However, few of these variables have been evaluated systematically. Therefore, a comprehensive meta-analysis would allow for evaluation of all of these covariates as potential determinants of the overall effect size.

\section{References $^{1}$}

[1] Centers for Disease Control and Prevention [CDC], National Center for Injury Prevention and Control (September 19, 2012). Injury and Prevention Control: Traumatic Brain Injury. Retrieved from http://www.cdc.gov/traumaticbraininjury/statistics.html

[2] Allen, B. J., \& Gfeller, J. D. (2011). The immediate post-concussion assessment and cognitive testing battery and traditional neuropsychological measures: A construct and concurrent validity study. Brain Injury, 25(2), 179-191.

[3] Wu, X., Mao, H., Liu J., Xu, J., Cao, J., Gu, X., \& Cui, G. (2013). Dyanmic changes of SGK expression and its role in neuron apoptosis after traumatic brain injury. International Journal of Clinical and Experimental Pathology, 6(7), 1282-1293.

[4] McNally, K. A., Bangert, B., Dietrick, A., Nuss, K., Rusin, J., Wright, M., Taylor, H. G., \& Yeates, K. O. (2013). Injury versus noninjury factors as predictors of postconcussive symptoms following mild traumatic brain injury in children. Neuropsychology, 27(1), 1-12.

[5] Brain Injury Association of America [BIAA] (2013). Living With Brain Injury: Treatment. Retrieved from: http://biausa.fyrian.com/brain-injury-treatment.htm\#treatment continuum

\footnotetext{
${ }^{1}$ References marked with an asterisk indicate studies included in the meta-analysis.
} 
[6] D'Alessandro, G., Gallo, F., Vitaliano, A., Del Col, P., Gorraz, F., De Cristofaro, R., \& Boaretto, G. (2010). Prevalence of stroke and stroke-related disability in Valle d'Aosta, Italy. Neurological Sciences, 31, 137-141.

[7] Centers for Disease Control and Prevention [CDC], National Center for Chronic Disease Prevention and Health Promotion (September 19, 2012). Division for Heart Disease and Prevention:Stroke-from http://www.cdc.gov/stroke/facts.htm

[8] Persson, H. C., Parziali, M., Danielsson, A., \& Sunnerhagen, K. S. (2012). Outcome and upper extremity function within 72 hours after first occasion of stroke in an unselected population at a stroke unit. A part of the SALGOT study. BMC Neurology, 12(162), 1-6.

[9] Bakheit, A. M. O., Shaw, S., Carrington, S., \& Griffiths, S. (2007). The rate and extent of improvement with therapy from the different types of aphasia in the first year after stroke. Clinical Rehabilitation, 21(10), 941-949.

[10] Beveridge, M. E. L., \& Bak, T. H. (2011). The languages of aphasia research: Bias and diversity. Aphasiology, 25(12), $1451-1468$.

[11] Glymour, M. M., Yen, J. J., Kosheleva, A., Moon, J. R., Capistrant, B. D., \& Patton, K. K. (2012). Elevated depressive symptoms and incident storke in Hispanic, African-American, and White older Americans. Journal of Behavioral Medicine, $35,211-220$.

[12] Siniscalchi, A., Gallelli, L., Labate, A., Malferrari, G., Palleria, C., Sarro, G.D. (2012). Post-stroke movement disorders: Clinical manifestations and pharmacological management. Current Neuropharmacology, Sep; 10(3): 254-262.

[13] Lerdal, A., Bakken, L. N., Rasmussen, E. F., Beiermann, C., Ryen, S., Pynten, S., Drefvelin, Å. S., Dahl, A. M., Rognstad, G., Finset, A., Lee, K. A., \& Kim, H. S. (2011). Physical impairment, depressive symptoms and pre-stroke fatigue are related to fatigue in the acute phase after stroke. Disability and Rehabilitation, 33(4), 334-342.

[14] *Särkämö, T., Tervaniemi, M., Laitinen, S., Forsblom, A., Soinila, S., Mikkonen, M., Autti, T., Silvennoinen, H. M., Erkkilä, Laine, M., Peretz, I., \& Hietanen, M. (2008). Music listening enhances cognitive recovery and mood after middle cerebral artery stroke. Brain, 131, 866-876.

[15] Conklyn, D., Novak, E., Boissy, A., Bethoux, F., \& Chemali, K. (2012). The effects of modified melodic intonation therapy on nonfluent aphasia: A pilot study. Journal of Speech, Language, and Hearing Research, 55, 1463-1471.

[16] Kirmess, M., \& Maher, L. M. (2010). Constraint induced language therapy in early aphasia rehabilitation. Aphasiology, 24(6-8), 725-736.

[17] Turkstra, L. S. (2008). Conversation-based assessment of social cognition in adults with traumatic brain injury. Brain Injury, 22(5), 397-409.

[18] Fecteau, S., Agosta, S., Oberman, L., \& Pascual-Leone, A. P. (2011). Brain stimulation over Broca's area differentially modulates naming skills in neurotypical adults and individuals with Asperger's syndrome. European Journal of Neuroscience, 34, 158-164.

[19] Fridriksson, J., Hubbard, H. I., Hudspeth, S. G., Holland, A. L., Bonilha, L., Fromm, D., \& Rorden, C. (2012). Speech entrainment enables patients with Broca's aphasia to produce fluent speech. Brain, 135, 3815-3829.

[20] Kemmerer, D. (2012). The cross-linguistic prevalence of SOV and SVO word orders reflects the sequential and hierarchical representation of action in Broca's area. Language and Linguistics Compass, 6(1), 50-66.

[21] Konrad, A., Vucurevic, G., Musso, F., \& Winterer, G. (2012). VBM-DTI correlates of verbal intelligence: A potential link to Broca's area. Journal of Cognitive Neuroscience, 24(4), 888-895.

[22] Josephs, K. A., Duffy, J. R., Strand, E. A., Machulda, M. M., Senjem, M. L., Master, A. V., Lowe, V. J., Jack, C. R., \& Whitwell, J. L. (2012). Characterizing a neurodegenerative syndrome: Primary progressive apraxia of speech. Brain, 135, 1522-1236.

[23] Poeppel, D., Emmorey, K., Hickok, G., \& Pylkkänen, L. (2012) Towards a new neurobiology of language. The Journal of Neuroscience, 32(41), 14125-14131.

[24] Meguro, K. (2012). Behavioral neurology in language and aphasia: From basic studies to clinical applications. The Indonesian Journal of Internal Medicine, 327-334.

[25] Cicerone, K. D., Dahlberg, C., Kalmar, K., Langenbahn, D. M., Malec, L. E., Berguist, T. F., et al. (2000). Evidence-based cognitive rehabilitation: Recommendations for clinical practice. Archives of Physical Medicine and Rehabilitation, 81, 1596-1615.

[26] Cicerone, K. D., Dahlberg, C., Malec, J. F., Langenbahn, D. M., Felicetti, T., Kneipp, S., et al. (2005). Evidence-based cognitive rehabilitation: Updated review of the literature from 1998 through 2002. Archives of Physical Medicine and Rehbailitation, 86, 1681-1692.

[27] Robey, R. U. (1998). A meta-analysis of clinical outcomes in the treatment of aphasia. Journal of Speech, Language, and Hearing Research, 41(1), 172-187.

[28] Rohling, M. L., Faust, M. E., Beverly, B., Demakis, G. (2009). Effectiveness of cognitive rehabilitation following acquired brain injury: A meta-analytic re-examination of Cicerone et al.'s $(2000,2005)$ systematic reviews. Neuropsychology, 23(1), 20-39.

[29] Wisenburn, B., \& Mahoney, K. (2009). A meta-analysis of word-finding treatments for aphasia. Aphasiology, 23(11), 1338-1352.

[30] Elliot, M., \& Parente, R. (in press). Efficacy of Memory Rehabilitation Therapy: A Meta-Analysis of TBI and Stroke Cognitive Rehabilitation Literature. Brain Injury. Early On-Line-DOI:10.3109/02699052.2014.934921, http://informahealthcare.com/bij, ISSN: 0269-9052 (print), 1362-301X (electronic)

[31] Bernard, R. (2010). The Efficacy of Cognitive Rehabilitation for Attention Deficits in Sustained Traumatic Brain Injury and Stroke: A Meta-Analytic Review (Unpublished master's thesis). Towson University, Maryland.

[32] Wilkinson, L., \& the APA Task Force on Statistical Inference. (1999). Statistical methods in psychology journals: Guidelines and explanations. American Psychologist, 54, 594-604.

[33] Durlak, J. A. (2009). How to select, calculate, and interpret effect sizes. Journal of Pediatric Psychology, 34(9), 917-928. 
[34] Rosenthal, R. (2002, August). Correlations, contrasts, and conceptual clarity. Paper presented at the Annual Meeting of the American Psychological Association, Chicago, IL.

[35] Quality Standards Subcommittee. American Academy of Neurology. Practice parameter: the management of concussion in sports. Neurology, 48: 581-585, 1997.

[36] Saur, D., \& Hartwigsen, G. (2012). Neurobiology of language recovery after stroke: Lessons from neuroimaging studies. Archives of Physical Medicine and Rehabilitation, 93(1, Suppl 1), S15-S25.

[37] Sohlberg, M. M., \& Mateer, C. A. (1986). Effectiveness of an attention-training program. Journal of Clinical and Experimental Neuropsychology, 9(2), 117-130.

[38] *Bakheit, A. M. O., Carrington, S., Griffiths, S., \& Searle, K. (2005). High scores on the Western Aphasia Battery correlate with good functional communication skills (as measured with the Communicative Effectiveness Index) in aphasic stroke patients. Disability and Rehabilitation, 27(6), 287-291.

[39] *Bakheit, A. M. O., Shaw, S., Barrett, L., Wood, J., Carrington, S., Griffiths, S., Searle, K., \& Koutsi, F. (2007). A prospective, randomized, parallel group, controlled study of the effect of intensity of speech and language therapy on early recovery from poststroke aphasia. Clinical Rehabilitation, 21, 885-894.

[40] *Braden, C., Hawley, L., Newman, J., Morey, C., Gerber, D., \& Harrison-Felix, C. (2010).
[41] *Chou, P., Chu, H., \& Lin, J. G. (2009). Effects of electroacupuncture treatment on impaired cognition and quality of life in Taiwanese stroke patients. The Journal of Alternative and Complementary Medicine, 15(10), 1067-1073.

[42] *Kersel, D. A., Marsh, N. V., Havill, J. H., \& Sleigh, J. W. (2001). Neuropsychological functioning during the year following severe traumatic brain injury. Brain Injury, 15(4), 283-296.

[43] *King, K. A., Hough, M. S., Walker, M. M., Rastatter, M., \& Holbert, D. (2006). Mild traumatic brain injury: Effects on naming in word retrieval and discourse. Brain Injury, 20(7), 725-732.

[44] *Meinzer, M., Djundja, D., Barthel, G., Elbert, T., \& Rockstroh, B. (2005). Long-term stability of improved language functions in chronic aphasia after constraint-induced aphasia therapy. Stroke, 36, 1462-1466.

[45] *Mumby, K. \& Whitworth, A. (2012). Evaluating the effectiveness of intervention in long-term aphasia post-stroke: The experience from CHANT (Communication Hub for Aphasia in North Tyneside). Journal of Language and Communication Disorders, 47(4), 398-412.

[46] *Snow, P., Douglas, J., \& Ponsford, J. (1998). Conversational discourse abilities following severe traumatic brain injury: A follow-up study. Brain Injury, 12(11), 911-935. 